\title{
Estudio anatómico mediante radiografía de zarigüeya común (Didelphis marsupialis) en zonas periurbanas de Medellín, Colombia
}

\author{
Anatomical X-ray study of common opossum (Didelphis marsupialis) in peri-urban \\ areas of Medellín, Colombia \\ Jessica Zapata Muñoz', Maribel Echeverry Hernández ${ }^{1}$, Sergio Andrés Correa ${ }^{1}$, \\ Cristian Ferney Esquivel, Daisy A. Gómez-Ruiz ${ }^{1}$, Ana Cristina Cadavid, \\ Ricardo Zambrano Valdés ${ }^{1,2}$
}

\section{Resumen}

\begin{abstract}
Este trabajo busca describir la morfología radiológica de la zarigüeya común (Didelphis marsupialis), estableciendo las bases para una correcta manipulación y manejo clínico adecuado. Los individuos fueron capturados con trampas Tomahawk cebadas en el corregimiento de Santa Elena, municipio de Medellín, Colombia. Se identificó el sexo y se verificó la posible presencia de crías en el marsupio (animales con crías fueron liberados). Se utilizó un protocolo anestésico basado en xilacina, tiletamina y zolazepam y se procedió a las tomas radiográficas lateral derecha, dorsoventral y ventrodorsal en el esqueleto axial (cráneo, tórax, abdomen y pelvis) y medio-laterales, y en vista anteroposterior y dorso-plantar y palmar en el esqueleto apendicular. Se encontró coincidencias con otros marsupiales en la presencia de la ossa marsupialia o hueso epipúbico, la fórmula vertebral y la ausencia de la patela; además, se confirma la presencia de estructuras como la bulla timpánica.
\end{abstract}

Palabras clave: diagnóstico imagenológico, radiología, anestesia, marsupial, bienestar animal

\footnotetext{
${ }^{1}$ Grupo GINVER, Facultad de Medicina Veterinaria, Corporación Universitaria Remington, Colombia

${ }^{2}$ E-mail:erzv2@hotmail.com
}

Recibido: 25 de octubre de 2020

Aceptado para publicación: 25 de abril de 2021

Publicado: 24 de agosto de 2021

CLos autores. Este artículo es publicado por la Rev Inv Vet Perú de la Facultad de Medicina Veterinaria, Universidad Nacional Mayor de San Marcos. Este es un artículo de acceso abierto, distribuido bajo los términos de la licencia Creative Commons Atribución 4.0 Internacional (CC BY 4.0) [https:// creativecommons.org/licenses/by/4.0/deed.es] que permite el uso, distribución y reproducción en cualquier medio, siempre que la obra original sea debidamente citada de su fuente original 
The aim of this study was to describe the radiological morphology of common opossum(Didelphis marsupialis), establishing the foundation for a correct manipulation and adequate clinical management. The individuals were captured with baited Tomahawk traps in the village of Santa Elena, municipality of Medellín, Colombia. The sex of the animals was identified and the possible presence of pups in the pouch was verified (animals with pups were released). An anesthetic protocol based on xylazine, tiletamine and zolazepam was used and the right lateral, dorsoventral and ventrodorsal X-rays were taken in the axial skeleton (skull, thorax, abdomen and pelvis) and medial-lateral, anteroposterior and dorsal, plantar and palmar in the appendicular skeleton. It was found coincidences with other marsupials in the presence of the ossa marsupialia or epipubic bone, the vertebral formula and the absence of the patella; Furthermore, the presence of structures such as the tympanic bulla is confirmed.

Key words: diagnostic imaging, radiology, anesthesia, marsupial, animal welfare

\section{INTRODUCCIÓN}

Didelphis marsupialis (D. marsupialis) es un marsupial que se caracteriza por una gruesa capa de piel, garras afiladas, bigotes largos, cola larga y anillo negro alrededor de ambos ojos, así como grandes orejas negras (Mohamed, 2018a). La cola es de tipo prensil, sus miembros son cortos y cada pata presenta cinco dedos, donde el primer dedo de los miembros posteriores es oponible, característico que les permite sujetar objetos y mejorar su habilidad trepadora (Cuartas-Calle y Arango, 2003). La fórmula dentaria de la especie es I 5/4, C 1/1, P 3/3, M 4/4, presentando una característica de interés para determinar la edad, debido a que su tercer molar carece de raíz en la etapa juvenil (Cuartas-Calle y Arango, 2003; Rueda et al., 2013).

La zarigüeya $D$. marsupialis es una especie que puede encontrarse fácilmente en zonas urbanas y periurbanas del Valle de Aburrá (Saldaña et al., 2019); sin embargo, sus poblaciones se encuentran amenazadas localmente por la falta de conocimiento y educación, además de la pérdida de hábitat y el atropellamiento vehicular (Delgado, 2007; Rueda et al., 2013; Cabrera et al., 2017).
Estudios previos han detallado la morfología del cráneo de D. marsupialis, buscando mejorar el abordaje clínico y terapéutico de esta especie describiendo los accidentes más relevantes y/o importantes (Mohamed, 2018b). Así mismo, otros estudios han utilizado la morfometría para identificar las diferencias interespecies de Didelphis (por ejemplo D. aurita y D. marsupialis, Cerqueira y Lemos, 2000). Se comprende el estudio radiológico como una herramienta para el diagnóstico clínico de los vertebrados y presenta una gran importancia en las ayudas diagnósticas a las que el médico veterinario del medio puede acceder (Owens y Biery, 1998). Incluyendo la información que se puede obtener del uso de la radiología como ayuda diagnóstica ante- $\mathrm{y}$ pos-mortem (Massad et al., 2016a,b; Gallego et al., 2009).

El presente trabajo aporta una descripción anatómica por medio de radiografía simple de una población de zarigüeyas en el corregimiento de Santa Elena (Medellín, Colombia), con el fin de describir la osteología, los accidentes óseos y las estructuras más llamativas o relevantes para la especie $D$. marsupialis. El objetivo del proyecto fue 
generar información acerca de la anatomía radiológica de D. marsupialis, con el fin de establecer puntos anatómicos de referencia para abarcar un diagnóstico oportuno y una correcta manipulación como herramienta de manejo veterinario y reconocimiento de las especies.

\section{Materiales y Métodos}

\section{Área de Estudio}

El estudio fue realizado en la clínica veterinaria de la Corporación Universitaria Remington ubicada en el corregimiento de Santa Elena, zona oriental del área urbana del municipio de Medellín, Colombia, sobre la cordillera central y a una altitud promedio de $2500 \mathrm{msnm}$. La zona limita por el norte con los municipios de Copacabana y Bello, al oriente con los municipios de Guarne y Rionegro, al occidente con el área urbana del municipio de Medellín y al sur con el municipio de Envigado. La zarigüeya común fue reportada en el km 9 Vía Santa Elena, lugar escogido para el trampeo. La zona presenta diversos tipos de cobertura vegetal y está rodeada por bosque natural fragmentado, pastos enmalezados, pastos arbolados y potreros (IDEAM, 2010).

\section{Captura de los Individuos}

Se emplearon tres trampas Tomahawk cebadas con sardinas, una mezcla de crema de maní y avena o frutas. Las trampas se colocaron en el suelo, en posibles sitios de paso, refugio y anidación de los marsupiales D. marsupialis (vegetación, fuentes de agua $\mathrm{y}$ alimento). Las trampas fueron colocadas entre las 16:00 y 18:00, evitando los días de lluvias, y se recogían al día siguiente entre las 07:00 y las 08:00.

Las trampas con captura de las zarigüeyas fueron cubiertas con una manta oscura para evitar estrés del animal a estímulos visuales y de luz debido a sus hábitos noctur- nos. Además, los integrantes del equipo emplearon elementos de protección personal como guantes de látex, guantes de carnaza y, tapabocas. Inmediatamente a la captura se verificó si las zarigüeyas tenían crías en el marsupio, teniendo en cuenta que su gestación es de 12-13 días y las crías terminan su desarrollo dentro del marsupio (Rueda et al., 2013). Los individuos con crías fueron dejados en libertad en el mismo lugar donde fueron capturados.

\section{Preparación para el Estudio Radiológico}

Dadas las dimensiones corporales de esta especie (individuos pequeños) y en la mayoría de los casos propensos a movimientos defensivos, tanto como de rechazo como de agresión, suele ser difícil su correcto posicionamiento y, por lo tanto, la obtención de una imagen de buena calidad. Por estas razones se justifica la realización de un protocolo de anestesia general a corto plazo que permita disminuir el estrés del individuo, posicionarlo correctamente, evitar repetir tomas y, por tanto, disminuir la sobreexposición radiológica (Krautwald-Junghanns et al., 2010).

Para realizar el protocolo de anestesia, el animal fue pesado dentro de una bolsa de tela, luego el médico veterinario radiólogo y el médico veterinario anestesista procedieron con el protocolo de anestesia establecido: Xilacina $2 \%$, (Hidrocloruro) $1 \mathrm{mg} / \mathrm{kg}$ y Zoletil 50\% (Tiletamina $25 \mathrm{mg}$ y Zolazepam $25 \mathrm{mg}) 3 \mathrm{mg} / \mathrm{kg}$. Una vez el individuo permitió la manipulación bajo el efecto de la anestesia, se procedió a la evaluación del animal, buscando posibles heridas, ectoparásitos, lesiones consolidadas o alteraciones relevantes. Se tomaron las medidas biométricas estándar para mamíferos (largo cabeza a cola, largo de cola, largo de las orejas, largo de miembros anteriores y posteriores y altura a la cruz), fueron pesados, y se determinó si el individuo se encontraba apto para continuar con el procedimiento. El animal, luego del procedimiento radiológico fue devuelto a la 
trampa Tomahawk para su recuperación anestésica; dejando la trampa nuevamente cubierta con una manta y en oscuridad.

Adicionalmente, para una pronta recuperación se suministró oralmente agua con azúcar por medio de jeringa. Los individuos fueron monitoreados pos-anestesia durante cuatro horas, buscando asegurar que el animal no presentara signos de hipotermia, ataxia o comportamientos inadecuados. Una vez que los animales se recuperaron de la anestesia fueron liberados en el mismo lugar de la captura.

\section{Estudios Radiológicos}

La radiografía busca, en términos generales, la representación y clasificación topográfica de estructuras anatómicas por medio de una imagen bidimensional, y dicha representación solo es posible cuando se realiza un estudio en al menos dos proyecciones (Krautwald-Junghanns et al., 2010). Para el caso de mamíferos exóticos, los principios radiológicos siguen los mismos principios usados en otros pequeños animales (Kealy et al., 2010). Es importante resaltar que las radiografías de cuerpo completo aumentan la probabilidad de subdiagnóstico u omisión. Estudios previos recomiendan la realización de radiografías según el segmento corporal de interés (Krautwald- Junghanns et al., 2010).

Los estudios radiológicos se realizaron con un equipo Diagnostic X-Ray Unit modelo VET RAY 4 O (USA) y un digitalizador de imagen Digital Flat Panel Detector (iRay Tecnhnology) modelo Venu 1417v-PSI. El posicionamiento del paciente se realizó con cinta adhesiva o micropore y los estudios se iniciaron con posicionamientos convencionales como la radiografía Lateral derecha (Lder), particularmente en el esqueleto axial, en regiones como el cráneo, tórax, abdomen $y$ pelvis, y en un segundo plano se tomaron vistas DorsoVentral o VentroDorsal (DV o VD). En el esqueleto apendicular se realiza- ron tomas MedioLaterales (ML) y en segundo plano en vista Anteroposterior (AP) o DorsoPlantar / Palmar (DP), siguiendo las recomendaciones de vistas estándar del examen radiológico rutinario, tanto como las abreviaciones recomendadas para las mismas (Kealy et al., 2010). La calibración del equipo y/o el cálculo de kilovoltaje y miliamperaje se basaron en la regla de Santes, la cual dicta que el valor resultante de la medición de la estructura a radiografiar (en centímetros), es multiplicada por dos (2) y se le suman 30, 40 o 50 como constantes para abdomen, hueso y tórax, respectivamente (Regla resultante: $\mathrm{Kv}=\mathrm{cm} \times 2+(30)$, en el caso del abdomen). $\mathrm{Al}$ obtener la imagen se procedió a la descripción de la topografía radiológica ósea y de estructuras anatómicas más relevantes de individuos de D. marsupialis y se comparó con la bibliografía revisada.

\section{Resultados}

Se capturaron siete individuos $D$. marsupialis (4 hembras, 3 machos), dos de los cuales fueron liberados pues tenían crías en su marsupio.

\section{Cráneo}

La descripción de la anatomía del cráneo se realizó mediante vistas lateral derecha (L-der) (Figura 1A,B), donde se puede apreciar el segmento vertebral cervical, y las vistas dorso-ventrales (DV) (Figura 1C,D). D. marsupialis presenta incisivos cortos y cónicos, caninos largos, desarrollados, y de aspecto agudo al igual que los premolares y molares.

\section{Tórax}

Se presentan las vistas dorso ventral (DV) donde se detallan las estructuras de la columna toráxica y el tórax (Figura 2A,B) la vista lateral derecha (L-der) mostrando la región toraco abdominal (Figura 2C). 

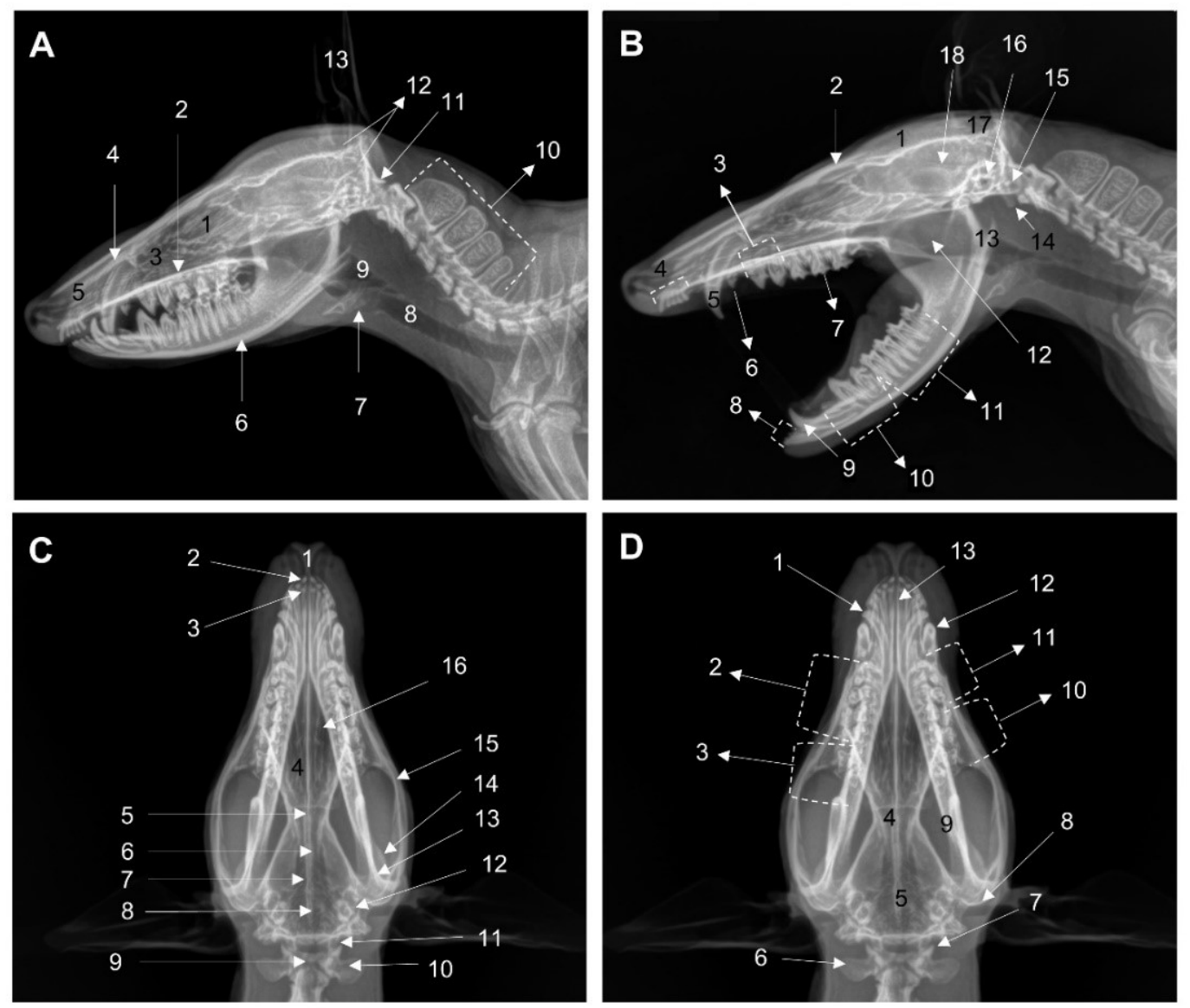

Figura 1. Vistas del cráneo. A. Vista L-der 1. Etnoturbinas, 2. Hueso palatino, 3. Cavidad nasal, 4. Hueso nasal, 5. Nasoturbinas, 6. Mandíbula, 7. Huesos hioideos, 8. Tráquea, 9. Laringe, 10. Segmento vertebral cervical, 11. Atlas (C1), 12. Cresta sagital, 13 Pabellón aurícular. B. Vista L-der 1. Hueso parietal, 2. Hueso frontal, 3. Premolares II, III, 4. Incisivos, 5. Canino, 6. Premolar I, 7. Molar, 8. Incisivos, 9. Canino, 10. Premolares I, II, III, 11. Molares, 12. Rama vertical de la mandíbula, 13. Apófisis condilar, 14. Apófisis yugular, 15. Cóndilos del occipital, 16. Bulla timpánica, 17. Hueso occipital, 18. Hueso temporal. C. Vista DV 1. Narina, 2. Incisivo Superior, 3. Incisivo inferior, 4. Hueso palatino, 5. Hueso vómer, 6. Hueso preesfenoides, 7. Hueso basiesfenoides, 8. Hueso basioccipital, 9. Apófisis odontoides de C1, 10. Atlas (C1), 11. Cóndilos del occipital, 12. Bulla timpánica, 13. Ángulo mandibular, 14. Apófisis coronoides de la mandíbula, 15, Hueso cigomático, 16. Cavidad nasal. D. Vista DV 1. Canino inferior, 2. Premolares inferiores I, II, III, 3. Molares inferiores I, II, III, IV, 4. Coanas, 5. Hueso basioccipital, 6. Atlas (C1), 7. Cóndilos del occipital, 8. Apófisis condilar, 9. Rama horizontal de la mandíbula, 10. Molares superiores I, II, III, IV, 11. Premolares superiores I, II, III, 12. Canino superior, 13. Nasoturbinas 

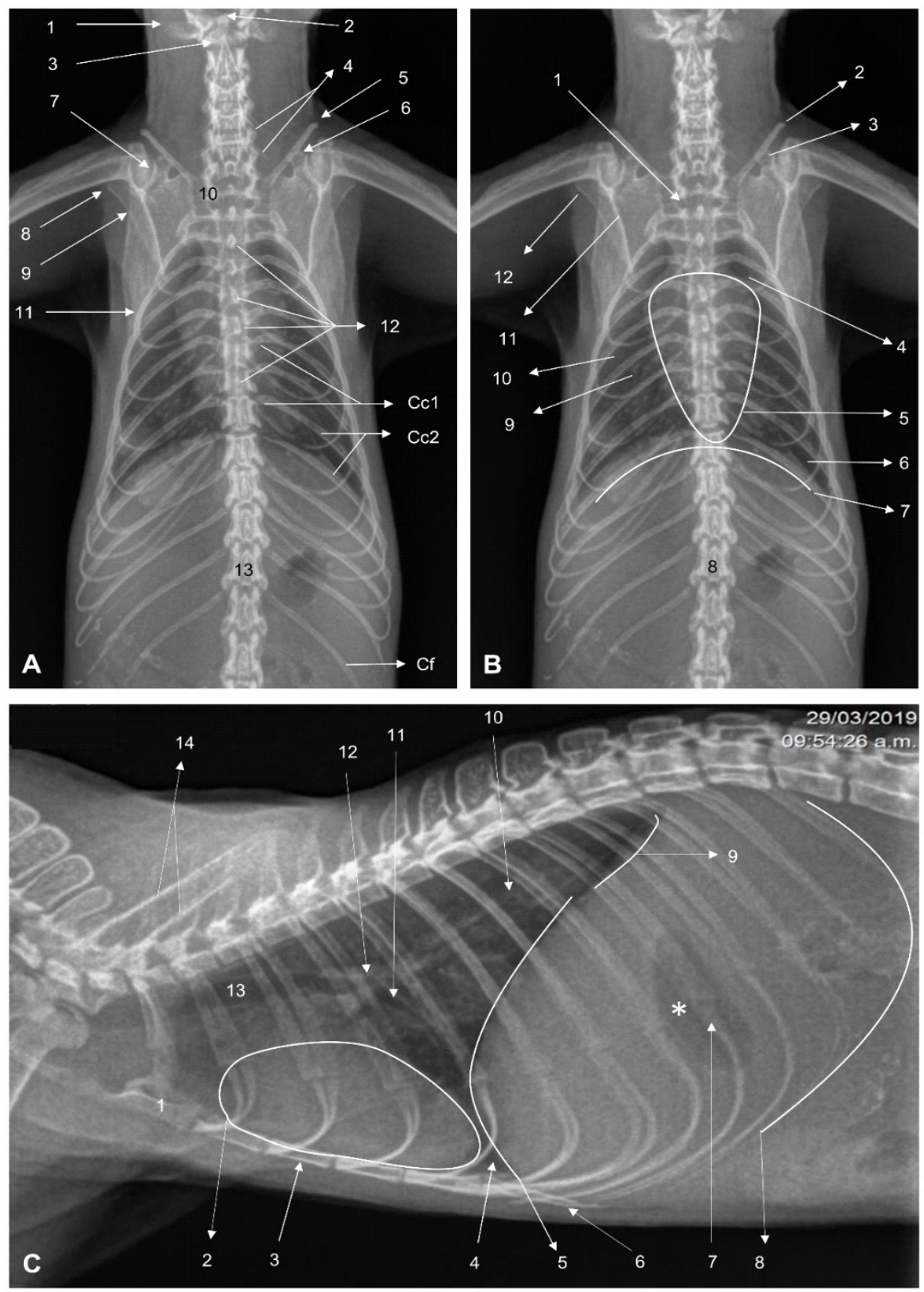

Figura 2. Vistas del tórax. A. Vista DV de la columna toráxica. 1. Alas del atlas, 2. Apófisis odontoides, 3. Apófisis espinosa de C2, 4. Apófisis transversas, 5. Clavícula, 6. Apófisis coracoides, 7. Borde glenoideo, 8. Acromion, 9. Espina de la escápula, 10. Vértebra toráxica 1 (T1), 11. Cuerpo de la costilla 3, 12. Apófisis espinosas de vértebras toráxicas, 13. Vertebra toráxica 13 (T13), Cc1. Cabeza de costilla, Cc2. Cuerpo de las costillas 8 y 9, Cf. Costilla flotante. B. Vista DV del tórax. 1. Vértebra toráxica 1, 2. Clavícula, 3. Apófisis coracoides, 4. Lóbulo craneal del pulmón, 5. Silueta cardiaca, 6. Lóbulo caudal del pulmón, 7. Diafragma, 8. Vértebra toráxica 13, 9. Corte longitudinal de bronquio, 10. Corte transversal de bronquio, 11. Espina de la escápula, 12. Acromion. C. Vista L-der. toraco abdominal, 1. Manubrio del esternón, 2. Silueta cardiaca, 3. Esternebra, 4. Lóbulo accesorio aireado, 5. Cúpula diafragmática, 6. Apófisis xifoideo, 7. Cartílago costal, 8. Arco costal, 9. Pilar diafragmático, 10. Corte transversal de bronquio, 11. Corte longitudinal de bronquio principal, 12. Carina, 13. Tráquea, 14. Espinas de la escápula, ${ }^{*}$ Silueta gástrica 
En la vista DV del tórax se evidencia una silueta cardiaca centrada y apoyada sobre el esternón, ocupando el espacio entre las costillas 1 a 2 y el espacio entre las costillas 4 y 5 . Sería posible evidenciar la silueta cardiaca derecha levemente dilatada, gracias a la presencia de dos apéndices en la aurícula derecha; así mismo, esta silueta puede verse separada del diafragma por el lóbulo accesorio (Figura 2A). Se muestra la inserción de los pilares del diafragma relacionada a la vértebra toráxica 12 .

\begin{abstract}
Abdomen
En la región del abdomen mediante la vista lateral derecha (L-der), se puede identificar algunas estructuras óseas y algunos órganos (Figura 3A), entre ellas una característica diferenciadora de los marsupiales con los mamíferos que es la presencia de huesos epipúbicos. En la Figura 3A se observa contenido alimenticio a nivel estomacal y la presencia de gas, así como material granular en el espacio intraluminal compatible en el espacio intraluminal con heces en colon. Las vísceras no se pueden distinguir fácilmente debido a la ausencia de resultados de tejido adiposo abdominal en siluetas positivas de vísceras adyacentes, camuflando sus superficies serosas. El colon domina la imagen radiográfica y, sumado al ciego, se sobreponen sobre los órganos en la cavidad, lo que en adición a la cantidad de grasa, disminuye la correcta visualización de estructuras.
\end{abstract}

\section{Columna y Cola}

La región de la columna cérvicotoráxica (Figura 3B y la región toraco-lumbar (Figura 3C) de presentan mediante vistas laterales, en tanto que la cadera (Figura 4A,B) y la cola (Figura 4C,D) mediante vista ventro dorsal.

En las vistas sobresale la morfología más larga de las apófisis espinosas de las vértebras cervicales, las cuales a su vez contrastan con la agudeza de las apófisis dorsales de las primeras vértebras toráxicas (Figura 3B,C). El axis (C2) presenta una apófisis espinosa que sobresale del atlas $(\mathrm{C} 1)$ y que en la vista DV se evidencia de forma triangular sobreponiéndose sobre C1 (Figura 3B). En esta vista se diferencias las apófisis odontoides de C2 (Figura 2A). Las vértebras lumbares llevan una apófisis transversa (Figura 3C).

En la cola, las apófisis transversas son visibles, lo que se emparejan adyacentes a las articulaciones vertebrales de las vértebras caudales medias (Figura 4B). Las apófisis transversas no son visibles caudalmente, aunque los arcos hemáticos (HA) persisten hasta el final de la cola (Figura 4D.

\section{Miembro Anterior (MA)}

Se presenta de manera general la disposición del miembro anterior en una vista lateral derecha (Figura 5A) y una descripción de la anatomía ósea en posición dorso palmar (DP) donde se observan los cinco dígitos (Figura 5B). En cuatro de ellos (2, 3, $4,5)$ se encuentran tres huesos de las falanges $3,4,5$, mientras que el dígito 1 presenta dos falanges. Finalmente se presenta en detalle la articulación metacarpiana en vista dorso palmar (Figura 5C).

La cresta supinadora (Figura 5A) está presente en la cara caudal del húmero distal. El extremo distal del radio y el cúbito forman la articulación antebraquiocarpiana con la fila proximal de los huesos del carpo (Figura 5A). $\mathrm{El}$ hueso carpiano intermedio es pequeño. La fila distal de los huesos del carpo comprende los huesos del carpo primero, segundo, tercero y cuarto (I, II, III, IV) en el extremo distal de cada hueso metacarpiano (Figura 5C).

En los miembros anteriores llama la atención la presencia de un área radiolúcida en la cara medial de la epífisis proximal del húmero y dorsal al cóndilo medial, la cual se confirmó en vistas radiológicas complementarias (Figuras 5B y 6A). Además, se observa la presencia de osteofitos, asimetría de la 

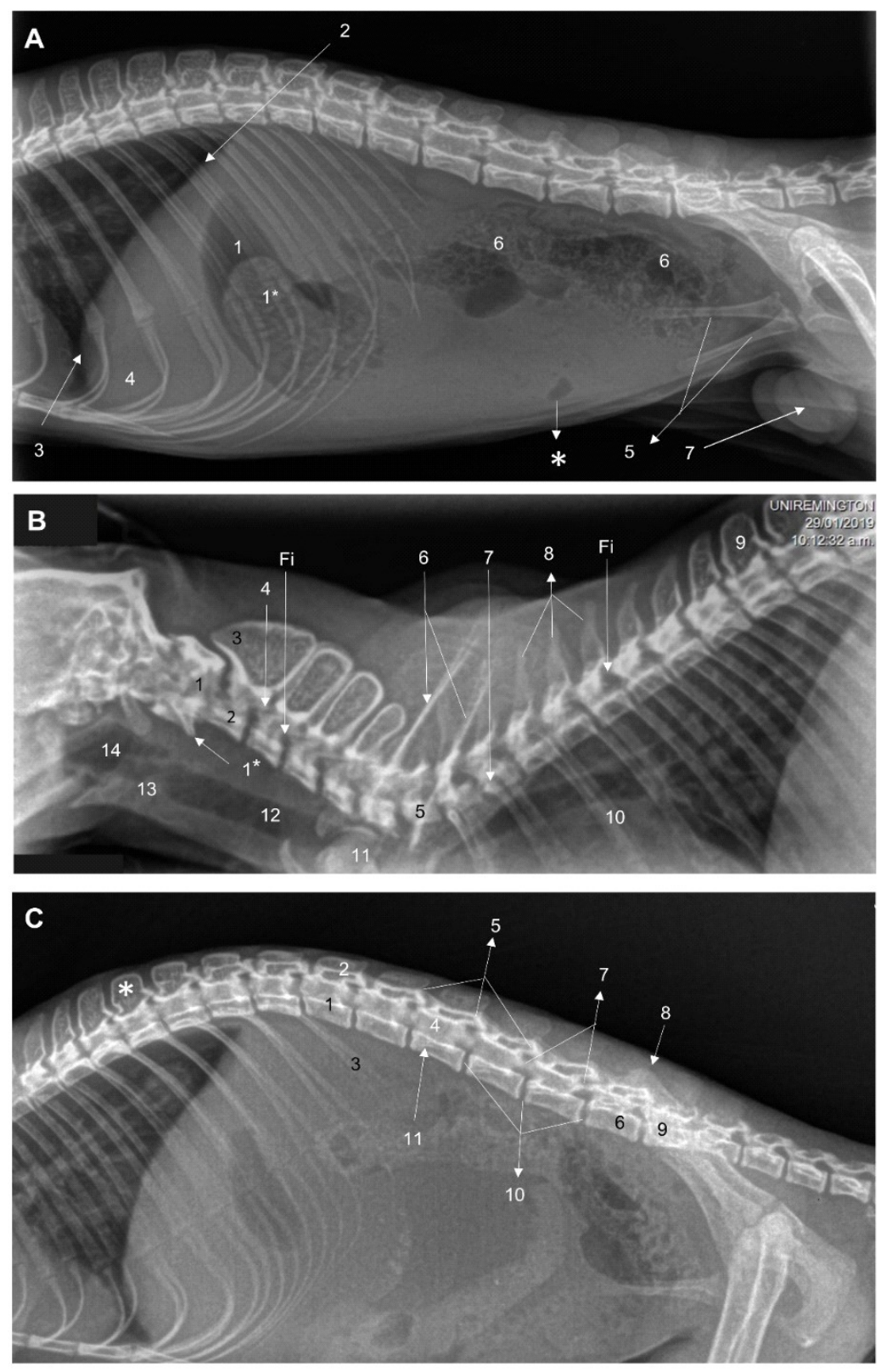

Figura 3. Vistas latero derecha de columna y abdomen A. Vista L-der del abdomen. 1. Estómago, $1 *$. Contenido estomacal, 2. Pilar del diafragma, 3. Cúpula del diafragma, 4. Área de proyección de la silueta hepática, 5. Huesos epipúbicos (ossa marsupialia), 6. Colon, 7. Testículos, *. Corte trasversal de intestino con presencia de gas. B. Vista L-der de columna cérvico - toráxica. 1. Atlas $(\mathrm{C} 1), 1^{*}$. Ala del atlas, 2. Axis (C2), 3. Apófisis espinosa de $\mathrm{C} 2$, 4. Faceta articular dorsal caudal de C2, Fi. Foramen intervertebral, 5. Vértebra cervical 7 (C7), 6. Espinas de la escápula, 7. Cabeza de la segunda costilla, 8 Apófisis espinosa de vertebras toráxicas 3,4 y 5, 9. Apófisis espinosa de vertebra toráxica 9 (T9), 10. Tercera costilla, 11. Cabeza humeral, 12. Tráquea, 13. Laringe, 14. Faringe. C. Vista L-der de columna toraco - lumbar. * Apófisis espinosa de vértebra toráxica 11 (T11), 1. Vértebra lumbar 1 (L1), 2. Apófisis espinosa de L1, 3. Costilla flotante, 4. Canal medular, 5. Apófisis articulares caudales de las vértebras lumbares 3, 4 y 5, 6. Vértebra lumbar 6 (L6), 7. Apófisis accesoria de L5 y L4, 8. Ilion, 9. Sacro (S1), 10. Espacios vertebrales, 11. Apófisis transversa 

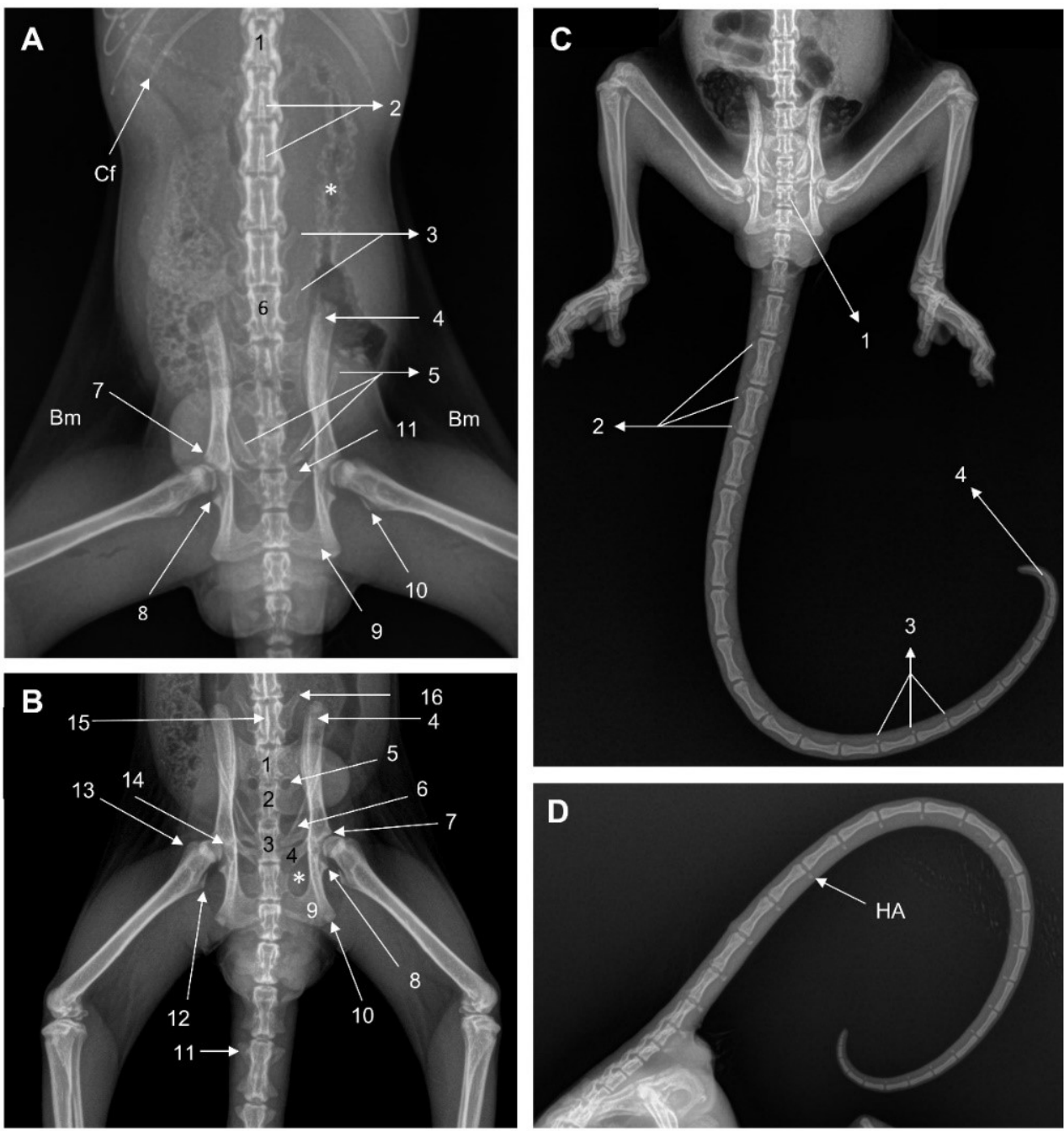

Figura 4. A. Vista DV de la región lumbo sacra, 1. Vértebra lumbar 1 (L1), 2. Apófisis espinosas de vértebras lumbares, 3. Apófisis transversas de vértebras lumbares, 4. Ilion, 5. Hueso epipúbico, 6. Vértebra lumbar 6(L6), 7. Borde craneal del acetábulo, 8. Borde caudal del acetábulo, 9. Isquion., 10. Trocánter menor, 11. Pubis, Cf. Costilla flotante, $\mathrm{Bm}$. Bolsa marsupial o marsupio, * corte longitudinal del intestino con presencia de gas. B. Vista VD de la cadera, 1. Vertebra sacra 1 (S1), 2. S2, 3. S3, 4. Pubis, 5. Agujero sacro, 6. Hueso epipúbico (ossa marsupialia) 7. Borde craneal del acetábulo, 8. Borde caudal del acetábulo, 9. Isquion, 10. Tuberosidad isquiática, 11. Apófisis transversa de vertebra coccígea, 12. Trocánter menor, 13. Trocánter mayor, 14. Acetábulo, 15. Apófisis espinosas de vértebras lumbares, 16. Apófisis transversa de L6, *. Agujero obturador. C. Vista VD, región de la cola, detalle de las vértebras coccígeas, 1 . Vértebra coccígea $1(\mathrm{Cc} 1), 2$. Apófisis transversas de vértebras coccígeas, 3 . Arcos hemáticos, 4. Última vértebra coccígea (Cc25). D. Detalle de los arcos hemáticos (HA) 

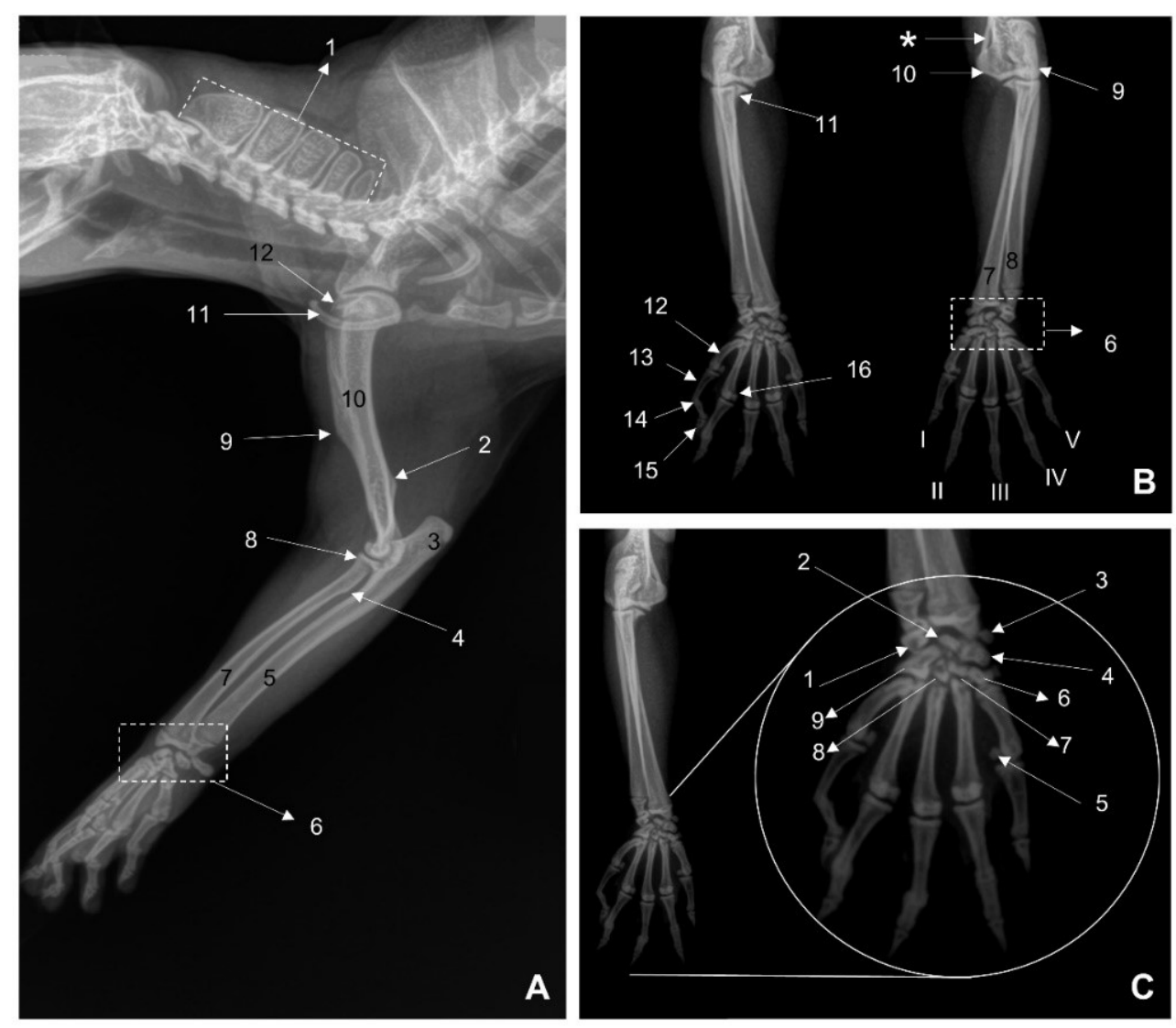

Figura 5. Vistas y detalles miembro anterior (MA). A. Vista L-der del MAI, 1. Segmento cervical, 2. Cresta supinadora, 3. Olécranon, 4. Tuberosidad radial, 5. Cúbito, 6. Huesos del carpo, 7. Radio, 8. Cabeza radial, 9. Tuberosidad deltoidea, 10. Húmero, 11. Clavícula, 12. Tubérculo mayor. B. Vista DP de miembros anteriores, 1. Dígito I, 2. Dígito II, 3. Dígito III, 4. Dígito IV, 5. Dígito V, 6. Huesos del carpo, 7. Radio, 8. Ulna, 9. Cóndilo lateral, 10. Cóndilo medial, 11. Cabeza radial, 12. Hueso metacarpiano 5, 13. Falange 1, 14. Falange 2, 15. Falange 3, 16. Huesos Sesamoideos. *. Estructura radio lucida muy bien definida. C. Vista DP de articulación metacarpiana del MAI, 1. Carpo ulnar, 2. Carpo intermedio, 3. Carpo accesorio, 4. Carpo radial, 5. Sesamoideos, 6. Hueso del carpo I, 7. Hueso del carpo II, 8. Hueso del carpo III, 9. Hueso del carpo IV

estructura y aumento del volumen, sugerente de proceso remodelativo crónico (Figura 6B) en uno de los pacientes estudiados.

\section{Miembro Posterior (MP)}

Se describe la anatomía ósea de la cadera y miembros posteriores en vista VD (Figura 7A), donde se puede observar las líneas de crecimiento en individuos juveniles.
Además, se observan detalles de la anatomía en vista medio lateral derecha del miembro posterior y la cadera (Figura 7B) y el detalle de la articulación de la rodilla (Figura 7C) en donde resalta la ausencia de patela. La articulación tarso tibial en vista VD (Figura 7D) muestra que el hueso talo y el calcáneo forman la base de apoyo de la articulación con la tibia y la fíbula. 

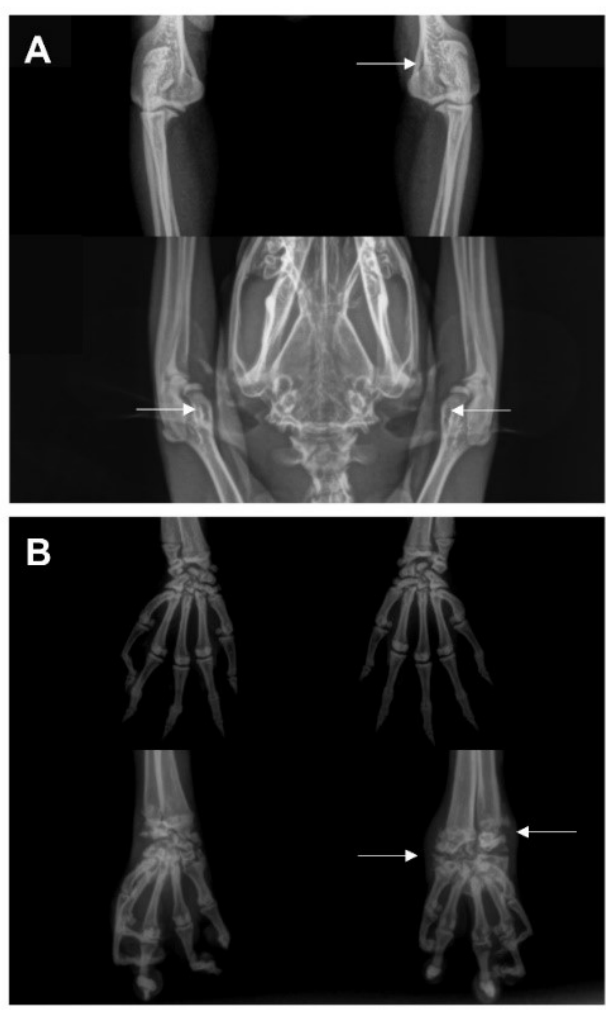

Figura 6. A. Vista comparativa de miembros anteriores (arriba) y vista VD de cráneo (abajo) con miembros anteriores en extensión (Flechas indican área radiolúcida). B. Vista AP comparativa de la región carpo radial (Flechas indican la presencia de osteofitos).

Son visibles las líneas fisiarias en cóndilos femorales y epífisis proximales de tibia y ulna en vista VD de MPD (Figura 7B,D). Así mismo, el sesamoideo lateral se denota más pequeño y desplazado hacia proximal, en comparación con pacientes de mayor edad. Los miembros son cortos y cada uno presenta cinco dedos, donde el primer dedo de los miembros posteriores es oponible, característica que les permite sujetar objetos y mejorar su habilidad trepadora.

\section{Discusión}

El presente estudio examinó de forma general y bajo protocolo de anestesia fijo la anatomía radiológica del cuerpo de individuos D. marsupialis, especialmente la morfología ósea. Además, se comparó la anatomía radiológica de estos individuos con la de otros marsupiales (como D. virginiana, D. albiventris y Pseudocheirus peregrinus de estudios reportados por Capello y Lennox (2013) y Vogelnest y Graeme, 2015). Otros estudios han descrito de manera general la anatomía del cráneo de D. marsupialis (Capello y Lennox, 2013; Vogelnest. y Graeme, 2015; Mohamed, 2018a,b), donde algunos concluyen que la anatomía craneana no difiere de otros marsupiales e incluso de otros pequeños mamíferos (Mohamed, 2018a).

El presente estudio coincide con Capello y Lennox (2013) y de Inamassu et al. (2017), con relación a la ausencia de la patela, estructura ausente también en otras especies de marsupiales (Reese et al., 2001; sin embargo, se difiere del estudio de Mohamed (2018a), quién indica ausencia de la bulla timpánica en $D$. marsupialis, toda vez que es una estructura diferenciable en las imágenes radiográficas (Figura 1B,C) y que ha sido reportada en marsupiales como $D$. virginiana (Capello y Lennox, 2013).

En los hallazgos radiológicos se pueden resaltar diversas observaciones. Por ejemplo, la fórmula vertebral de $D$. marsupialis coincide con lo reportado para otros marsupiales por Vogelnest y Graeme (2015), presentando 7 vértebras cervicales, 13 toráxicas, 6 lumbares, 2 a 3 sacras y para el caso de D. marsupialis con 25 vértebras coccígeas. Llama la atención la morfología más ancha y gruesa de las apófisis dorsales de la columna cervical, la cual podría expli- 

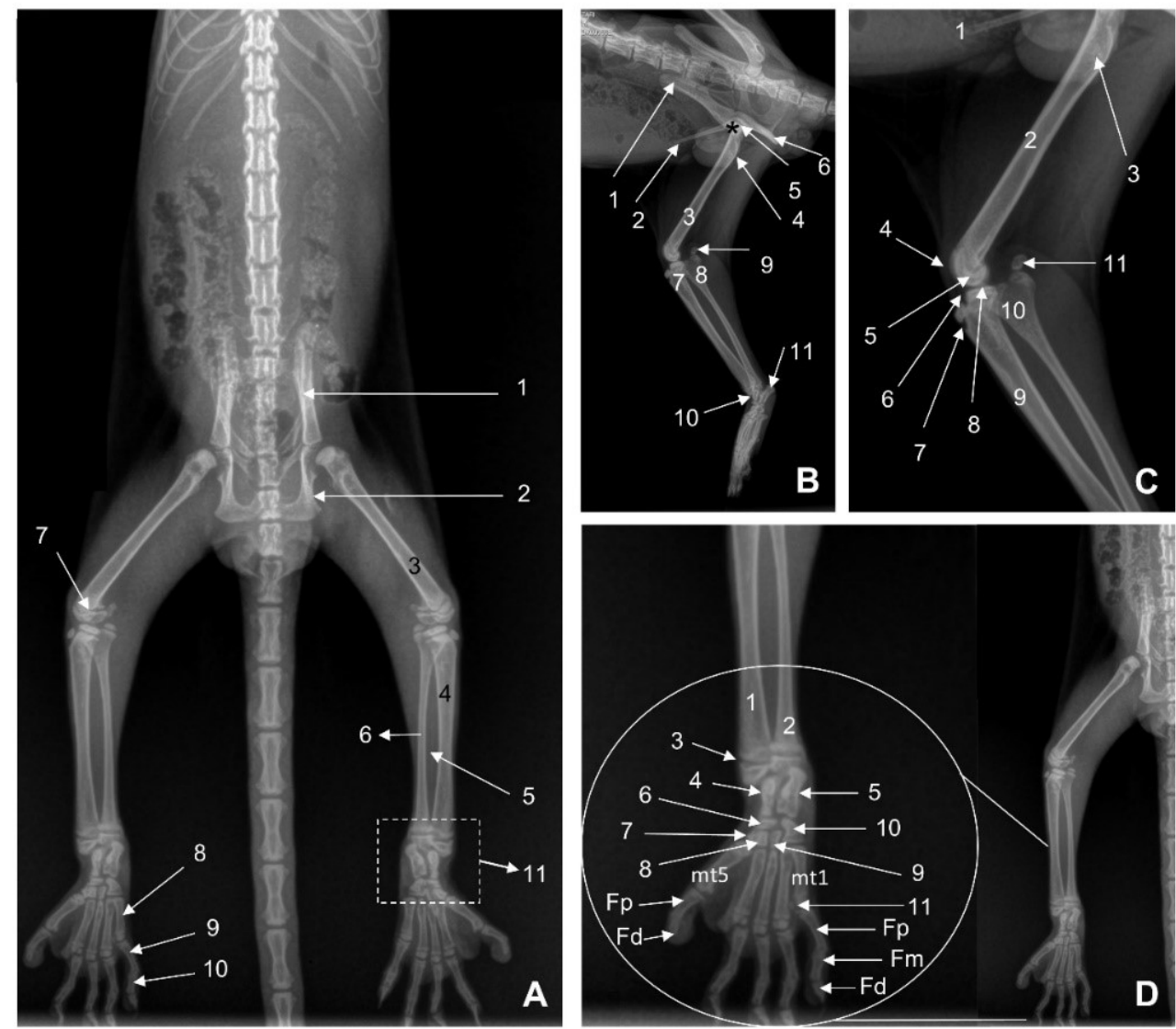

Figura 7. Vistas miembro posterior (MP). A. Vista VD de cadera y MP. 1. Ilion, 2. Isquion, 3. Fémur, 4. Tibia, 5. Espacio interóseo, 6. Fíbula, 7. Líneas de crecimiento en $D$. marsupialis juvenil, 8. Falange 1, 9. Falange 2, 10. Falange 3, 11. Huesos del tarso. B. Vista ML de MPD y rodilla; 1. Ilion, 2. Hueso epipúbico, 3. Fémur, 4. Trocánter menor, 5. Acetábulo, * Cabeza del fémur, 6. Isquion, 7. Tibia, 8. Fíbula, 9. Hueso Sesamoideo lateral, 10. Talo, 11. Tuberosidad calcánea. C. Detalle de articulación de la rodilla. 1. Hueso epipúbico, 2. Fémur, 3. Trocánter menor, 4. Tróclea del fémur, 5. Cóndilo femoral, 6. Faceta articular de la tibia, 7. Cresta de la tibia, 8. Espina de la tibia, 9. Tibia, 10. Fíbula, 11. Hueso sesamoideo lateral. D. Vista posterior - anterior oblicua del MPD, (a) Detalle de la articulación tarso tibial 1. Tibia, 2. Fíbula, 3. Línea fisiaria, 4. Talo, 5. Calcáneo, 6. Tarso central, 7. Tarsiano IV, 8. Tarsiano III, 9. Tarsiano II, 10. Tarsiano I, 11. Hueso Sesamoideo, Fp. Falange proximal, Fm. Falange media, Fd. falange distal

carse por su relación biomecánica con el tejido muscular adyacente; así mismo, y en contraste con la columna cervical, las apófisis dorsales de las vértebras toráxicas craneales toman la típica forma de espina (Figura 2C); donde la T11 cual presenta una apófisis espinosa que, similar a otros mamíferos, define una diferencia morfológica en las vértebras subsi- guientes (T12 y hacia caudal), en términos de apófisis espinosas progresivamente más pequeñas y apófisis transversas más prominentes. De otro lado, se confirmó la presencia de apófisis accesorias lumbares, las cuales se proyectan desde la superficie caudal de las vértebras (Vogelnest y Graeme, 2015). 
Con relación a la técnica radiológica, se debe considerar la morfología de la columna como un factor de complicación. El mantenimiento de la clásica posición VD (particularmente en regiones como columna, tórax y abdomen) involucra contar con almohadillas o cojines que aseguren el individuo, mantengan su posicionamiento y que no afecten la imagen; caso contrario será difícil la alineación y consecución de una imagen diagnostica. Ante esto, para las radiografías del esqueleto axial (exceptuando el cráneo), se recomienda considerar la vista DV, la cual puede disminuir tanto la manipulación como el tiempo para la toma radiográfica.

Otra de las complicaciones que se presenta es el bajo contenido graso a nivel de tejidos blandos (particularmente abdomen) (Capello y Lennox, 2013), por lo que, según lo evidenciado en este estudio, se podría aumentar levemente el kilovoltaje (Kv) del equipo, aumentar ligeramente el tiempo de exposición y/o disminuir la distancia focal. Adicionalmente, para visualizar correctamente el tracto gastrointestinal, se debe utilizar sustancias de contraste positivo como las suspensiones de sulfato de bario (Capello y Lennox, 2013).

D. marsupialis, al igual que en otros marsupiales como Pseudocheirus peregrinus, tienen manos de agarre con los dos primeros dígitos oponibles a los tres restantes (Vogelnest y Graeme, 2015), lo que les confiere una alta capacidad prensil tanto en sus miembros anteriores como posteriores. En estos se presenta un dedo lateral más corto, contrario a la presentación general de otros mamíferos, en los que el dedo más corto está ubicado hacia medial (Figuras $5 \mathrm{C}$ y 6D).

Es importante el establecimiento de la morfología radiológica normal del esqueleto de estos individuos en el contexto de salud animal, puesto que, como todos, están expuestos y sometidos a diferentes estímulos, algunos de ellos patológicos, capaces de generar cambios notorios en una ayuda diagnóstica tan de fácil acceso como lo sería la radiología simple (Figura 6B). El estudio es un aporte para la construcción de material académico de apoyo en la formación de médicos veterinarios tanto como para la práctica clínica en medicina de fauna silvestre.

\section{Conclusiones}

- El protocolo de anestesia implementado fue adecuado para D. marsupialis y puede usarse para casos de diagnóstico de segmentos corporales por medio de radiografía simple.

- El protocolo de sujeción y manipulación al momento de la radiografía fue acertado, disminuyendo el tiempo de exposición y evitando la sobreposición de las manos de los operadores, situación que puede complicar el diagnóstico en algunos casos de traumatismo apendicular distal.

- La técnica radiológica fue exitosa en términos de brindar imágenes de calidad diagnóstica y académica.

\section{Agradecimientos}

A la Corporación Universitaria Remington por la financiación del proyecto mediante la convocatoria Interna de Semilleros 2018. Al personal de la sede Santa Elena por su apoyo en la logística del proyecto.

\section{Literatura Citada}

1 Cabrera J, Galeano R, Mazabel R, Quintana D, Monsalve B. 2017. Evaluación del estado actual de zarigüeyas (Didelphis marsupialis) en tres zonas del valle de Aburrá. J Agric Anim Sci 6: 30-40. doi: 10.22507/jals.v6n1a3

2 Capello V, Lennox AM. 2013. Clinical radiology of exotic companion mammals. USA: Wiley-Blackwell. 653 p. 
3 Cerqueira R, Lemos B. 2000. Morphometric differentiation between Neotropical black-eared opossums, Didelphis marsupialis and D. aurita (Didelphimorphia, Didelphidae). Mammalia 64: 319-328. doi: 10.1515/mamm.2000.64.3.319

4 Cuartas-Calle C, Arango JM. 2003. Marsupiales, cenoléstidos e insectívoros de Colombia. Colombia: Edi Universidad de Antioquia. 223 p.

5 Delgado C. 2007. Muerte de mamíferos por vehículos en la vía del Escobero, Envigado (Antioquia), Colombia. Actu Biol 29: 229-233.

6 Gallego Rodríguez, Reysner-Tavera J, Aguirre JC. 2019. Fractura mandibular múltiple en una zarigüeya (Didelphis marsupialis), estudio radiológico forense. Rev Colomb Cienc Anim 11: 682. doi: 10.24188/recia.v11.$\mathrm{n} 1.2019 .682$

7 [IDEAM] Instituto de Hidrología, Meteorología y Estudios Ambientales. 2010. Leyenda Nacional de Coberturas de la Tierra. Metodología CORINE Land Cover adaptada para Colombia Escala 1:100.000. Bogotá DC: IDEAM. 72 p.

8 Inamassu LR, Mamprim MJ, Dadalto CR, Cavaletti FC, Mello MC, Schimming BC. 2017. Absence of bony patella in the white-eared opossum (Didelphis albiventris): morphology and diagnostic imaging. Anat Histol Embryol 46: 611-614. doi: 10.1111/ahe.12316

9 Kealy JK, McAllister H, Graham JP. 2010. Diagnostic radiology and ultrasonography of the dog and cat. $5^{\text {th }}$ ed. USA: Saunders Elsevier. 585 p.

10 Krautwald-Junghanns ME, Pees M, Reese S. 2010. Diagnostic imaging of exotic pets: birds-small mammals-reptiles. Germany: Schlütersche Verlagsgesellschaft. $453 \mathrm{p}$.

11 Massad RL, Brandão de Campos AC, Rodrigues MMR, Sousa RN. 2016a. Necropsia virtual em animais. Rev Acad
Ciênc Anim 14: 145-155. doi: 10.7213/ academica.14.2016.16

12 Massad RL, Rodrigues MMR, Missen TT, Jacinto RST, Eising T, Sousa RN. 2016b. Postmortem analysis of injuries by roadkill of a white-eared opossum (Didelphis albiventis) by radiographs and forensic necropsy - a virtopsy case report. J Vet Sci Technol 7: 282. doi: 10.4172/2157-7579.1000282

13 Mohamed R. 2018a. Anatomical and radiographic study on the skull and mandible of the common opossum (Didelphis marsupialis Linnaeus, 1758) in the Caribbean. Vet Sci 5: 44. doi: 10.3390/vetsci5020044

14 Mohamed R. 2018b. A descriptive morphometric approach of the skull and mandible of the common opossum (Didelphis marsupialis Linnaeus, 1758) in the Caribbean and its clinical application during regional anaesthesia. Vet Sci 5: 29. doi: 10.3390/vetsci5010029

15 Owens J, Biery D. 1998. Radiographic interpretation for the small animal clinician. Germany: Wyley.

16 Reese S, Pfuderer UR, Bragulla $\mathrm{H}$, Loeffler K, Budras KD. 2001. Topography, structure and function of the patella and the patelloid in marsupials. Anat Histol Embryol 30: 289-294. doi: 10.1046/j.1439-0264.2001.00334.x

17 Rueda MC, Ramírez GF, Osorio JH. 2013. Aproximación a la biología de la zarigüeya común (Didelphis marsupialis). Bol Cient Mus His Nat 17: 141-153.

18 Saldaña GI, Cadavid AC, Gómez-R $D A$. 2019. Abundancia relativa y patrones de actividad de Didelphis marsupialis en un área periurbana de Medellín, Colombia. Rev MVZ Córdoba 24: 73667371. doi: 10.21897/rmvz.1352

19 Vogelnest L, Graeme A. 2015. Radiology of Australians mammals. Australia: CSIRO Publishing. 307 p. 